\title{
Erythrocyte Autoantibody Positivity in Serological Cross-matching
}

\author{
Bulent Atik, Tugba Kula Atik, Hulya Duran, Ozgur Baykan, Tuba Ersal, Emine Avci, Emre Ispir and Bayhan Bektore \\ Faculty of Medicine, Department of Anesthesiology and Reanimation, Balıkesir University, Turkey
}

\begin{abstract}
Objective: To investigate the erythrocyte autoantibody positivity detected in the serological cross-matching (XM), and its possible effects on salient hemogram parameters.

Study Design: A descriptive study.

Place and Duration of Study: Balıkesir Atatürk City Hospital's Blood Transfusion Centre, Faculty of Medicine, Department of Anesthesiology and Reanimation, Balıkesir University, Turkey, from 2017 to 2018.

Methodology: Erythrocyte autoantibody positivity, which was detected in the traditional serological cross-matching for a pre-transfusion laboratory test were analysed retrospectively. Later, hemogram changes in the previous (no erythrocyte autoantibodies) and following (erythrocyte autoantibodies present) transfusions were investigated using statistical methods.

Results: Erythrocyte autoantibody positivity rate was $10.16 \%(342 / 3,365)$. There was no statistically significant difference in the increase of hemoglobin, hematocrit, and red blood cell between the period when erythrocyte autoantibodies were detected or not, $(p=0.27,0.13$, and 0.09 , respectively).

Conclusion: Erythrocyte autoantibodies positivity found on routine cross-match exmination, which must be considered together with parameters such as previous transfusion history, other pre-transfusion laboratory test results, and clinical presentation and management.
\end{abstract}

Key Words: Transfusion, Erythrocye autoantibody, Alloantibody, Hemogram, Cross-matching.

How to cite this article: Atik B, Atik TK, Duran H, Baykan O, Ersal T, Avci E, Ispir E, Bektore B. Erythrocyte Autoantibody Positivity in Serological Cross-matching. J Coll Physicians Surg Pak 2020; 30(05):485-489. DOI: https://doi.org/10.29271/jcpsp.2020.05.485.

\section{INTRODUCTION}

Erythrocyte autoantibodies react with intrinsic antigens found in erythrocytes. ${ }^{1}$ Therefore, although the association between their detection and pathological effects remains to be confirmed, they are considered clinically important.

Viral infections, inflammatory processes, malignancies, and medicines are the main causes of erythrocyte autoantibodies; with a history of transfusion being the major factor. ${ }^{1,2}$ Infections increase the ability of macrophages to phagocytose the antibody-coated erythrocytes; and medicines activate the suppression of the immune system by erythrocyte autoantibody formation via several mechanisms. ${ }^{1}$ Blood transfusion plays a crucial role in alloantibody formation; and promoting erythrocyte autoantibody formation through a decline in the T lymphocyte system and immune system along with an increased function of the B lymphocyte system. ${ }^{1,3}$

Blood group identification and cross-matching (XM) are mandatory and crucial measures to ensure the safety and effectiveness of a blood transfusion.

Correspondence to: Dr. Bülent Atik, Department of Anesthesiology and Reanimation, Faculty of Medicine, Ballkesir University, Turkey

E-mail: bulent_atik@yahoo.com

Received: January 20, 2020; Revised: April 07, 2020;

Accepted: May 10, 2020

DOI: https://doi.org/10.29271/jcpsp.2020.05.485
Serological XM, for compatibility of blood components for transfusion by observing the antigen-antibody reaction between blood componentand recipient blood in-vitro, is an essential pre-transfusion laboratory test. ${ }^{4}$ Erythrocyte autoantibody is reported to range between $0.05 \%$ to $30 \%{ }^{5-9}$ According to several studies, the rate of erythrocyte autoantibody positivity in hospitalised patients ranged between $1 \%$ and $15 \%{ }^{4,9}$ Erythrocyte autoantibody positivity detected in hospitalised patients is due to the established mutual interaction between the drugs and erythrocytes, and $\mathrm{ABO}$ blood type-incompatible transfusions such as platelets. ${ }^{5,10}$ The rate of erythrocyte autoantibody positivity was reported to range between $0.05 \%$ and $0.56 \%$ in general population. $^{5,11}$

Such a high rate of erythrocyte autoantibody detection rate raises the question of whether erythrocyte autoantibody positivity should be considered either as a marker of hemolytic anemia or as an incidental finding only.

In this study, the aim was to evaluate the erythrocyte autoantibody positivity, detected in the routine serological XM for a pre-transfusion laboratory test, and its possible effects on some hemogram parameters.

\section{METHODOLOGY}

The ABO and Rh blood group identification and serological XM for a pre-transfusion laboratory test of 3,365 blood recipients were identified using an automatic blood group identification and blood XM analyser (Across System, DiaPro, Turkey) in Balıkesir Atatürk City Hospital's Blood Transfusion Centre from 2017 to 2018. 
Table I: Study steps' demographic data.

\begin{tabular}{|c|c|c|c|c|c|c|c|c|c|}
\hline & \multirow{2}{*}{$\begin{array}{c}\text { Age } \\
\text { (Median) }\end{array}$} & \multicolumn{2}{|c|}{$\begin{array}{l}\text { Gender } \\
\%(n)\end{array}$} & \multicolumn{3}{|c|}{$\begin{array}{l}\text { Clinics } \\
\% \text { (n) }\end{array}$} & \multicolumn{3}{|c|}{$\begin{array}{c}\text { Degree of positivity } \\
\%(n)\end{array}$} \\
\hline & & Male & Female & IM & ICU & Surgery & +1 & +2 & +3 \\
\hline $\begin{array}{l}\text { FSS } \\
(n: 342)\end{array}$ & $\begin{array}{c}72 \pm 16.11 \\
(0-98)\end{array}$ & $\begin{array}{l}52.05 \\
(178)\end{array}$ & $\begin{array}{l}47.95 \\
(164)\end{array}$ & $\begin{array}{l}43.86 \\
(150)\end{array}$ & $\begin{array}{l}32.75 \\
(112)\end{array}$ & $\begin{array}{c}23.39 \\
(80)\end{array}$ & $\begin{array}{l}4.97 \\
(17)\end{array}$ & $\begin{array}{l}93.86 \\
(321)\end{array}$ & $\begin{array}{c}1.17 \\
(4)\end{array}$ \\
\hline $\begin{array}{l}\text { SSS } \\
(n: 78)\end{array}$ & $\begin{array}{c}72 \pm 13.20 \\
(18-87)\end{array}$ & $\begin{array}{c}55.13 \\
(43)\end{array}$ & $\begin{array}{c}44.87 \\
(35)\end{array}$ & $\begin{array}{c}29.49 \\
(23)\end{array}$ & $\begin{array}{c}47.44 \\
(37)\end{array}$ & $\begin{array}{c}23.08 \\
(18)\end{array}$ & $\begin{array}{c}6.41 \\
(5)\end{array}$ & $\begin{array}{c}92.31 \\
(72)\end{array}$ & $\begin{array}{c}1.28 \\
(1)\end{array}$ \\
\hline
\end{tabular}

FSS: First step of the study, SSS: Second step of the study, IM: Departments of internal medicine, ICU: Intersive care units.

Table II: Comparison between the pre- and post-transfusion values according to the presence of erythrocyte autoantibodies.

\begin{tabular}{|l|c|c|c|}
\hline \multirow{2}{*}{$\begin{array}{l}\text { Hemogram } \\
\text { parameters } \\
\mathbf{n}=\mathbf{7 8})\end{array}$} & \multicolumn{2}{|c|}{ Erythrocyte autoantibodies } & \multirow{2}{*}{ Podian (25\%-75\%) } \\
\cline { 2 - 4 } & $\begin{array}{c}\text { No } \\
\text { Median (25\%-75\%) }\end{array}$ & \\
\hline $\begin{array}{l}\mathrm{HGB} \\
(12-16 \mathrm{~g} / \mathrm{dL})\end{array}$ & $1.1(0.7-1.5) / 1$ unit & $1.1(0.7-1.7) / 1$ unit & 0.27 \\
\hline $\begin{array}{l}\mathrm{HCT} \\
(35 \%-45 \%)\end{array}$ & $3.2(1.8-4.1) / 1$ unit & $3.1(1.9-5.1) / 1$ unit & 0.13 \\
\hline $\begin{array}{l}\mathrm{RBC} \\
\left(4-5 \times 10^{6} / \mu \mathrm{L}\right)\end{array}$ & $0.4(0.2-0.5) / 1$ unit & $0.4(0.2-0.6) / 1$ unit & 0.09 \\
\hline $\begin{array}{l}\text { HGB: Hemoglobine, HCT: Hemotocrite, RBC: Red Blood Cells. The level of } \\
\text { significance was accepted as } p<0.05 .\end{array}$ \\
\hline
\end{tabular}

In 342 of these 3,365 patients, erythrocyte autoantibody positivity $(+1,+2,+3)$ was detected in the traditional serological $\mathrm{XM}$ in the $\mathrm{AC}$ (auto-control) well, applied in the routine. AC well is coated with anti-human-globulin, where the recipient plasma and the recipient RBC are combined. Eight of 342 patients, evaluated by XM, were incompatible also, which was performed by combining the recipient plasma and the donor RBC with anti-human-globulin. Therefore, these eight patients underwent direct antiglobulin testing (DAT) (Across Gel Monospecific Direct Coombs, DiaPro, Turkey), indirect antiglobulin testing (Across Gel Neutral/AHG, DiaPro, Turkey), antibody identification testing (Across Gel Neutral, Across Gel AHG, DiaPro, Turkey), and minor blood group identification testing (Across Gel Rh Phenotyping with Kell, DiaPro, Turkey). The tests were performed according to the manufacturer's instructions. As the first step of the study, 342 patients with erythrocyte autoantibody positivity, which was detected in the XM, were included in the study and were analysed retrospectively from the hospital's data processing system.

Seventy-eight of 342 patients had a history of RBC suspension transfusion in our hospital, previously. It was understood that there were no erythrocyte autoantibodies, which was detected in the serological XM applied in the previous transfusions of these 78 patients. As the second stage of the study, these 78 patients were included in the study. The patients who reacted, had coexistence of erythrocyte autoantibodies+alloantibodies, and the patients under the age of 18 , were excluded from the study. In these 78 patients, the number of components transfused between the periods when erythrocyte autoantibodies were dectected and were not detected was determined, and the changes in hemoglobin (HGB), hematocrit (HCT), and RBC were calculated for one unit, with the ratios compared using Wilcoxon signed-rank test. Data were analysed using statistical package for social sciences version 22.0 for Windows (SPSS
Inc., Chicago, Illinois, United States of America). Numerical data, such as age, were expressed as median (IQR). Categorical data, such as gender, erythrocyte autoantibody positivity, were expressed as percentages and proportions. Statistical analysis data are presented as median interquartile range $(I Q R)$, and $n(\%)$. In all calculations, the level of significance was accepted as $p<0.05$. Using the Kolmogorov-Smirnov test, measured parameters were evaluated as to whether the distribution was normal or abnormal. In groups showing abnormal distribution, the Wilcoxon signed-rank test was used.

\section{RESULTS}

Erythrocyte autoantibody positivity was $10.16 \%(342 / 3,365)$ within the specified period. The median age of the 342 patients was $72 \pm 16.11(0-98)$ years. Moreover, $52.05 \%$ (178) of the patients were males, and $47.95 \%$ (164) were females. A total of $43.86 \%(150), 32.75 \%(112)$, and $23.39 \%(80)$ of the patients were receiving treatment in departments of internal medicine, intensive care units, and departments of surgery, respectively. Considering the degree of positivity, $93.86 \%$ (321), 4.97\% (17), and $1.17 \%(4)$ of the patients exhibited a positive reaction strength of $+2,+1$, and +3 , respectively (Table I). In the 342 patients who had erythrocyte autoantibody positivity, which was detected in the $\mathrm{XM}$, erythrocyte alloantibody positivity was detected in $2.3 \%$ of the patients (8/342) and the erythrocyte autoantibody+alloantibody co-existed in $0.24 \%$ of the patients $(8 / 3,365)$. All erythrocyte alloantibodies were anti-Rh (three anti$\mathrm{E}$, three anti-c, one anti-e, one anti-C). Eight patients who had erythrocyte alloantibody positivity, also had monospecific (IgG) DAT positivity. Positive reaction strengths were +1 in five and +2 in three of these patients. The patients were again subjected to the serological XM with minor blood type-compatible RBC suspensions; subsequently, the compatible RBC suspensions were obtained, and transfusions were performed in all patients except one patient. The physician of the patient, whose compatible RBC suspension could not be obtained, cancelled the transfusion.

Three of the 342 patients who had erythrocyte autoantibody positivity, which was detected in the serological XM, had mild transfusion reaction. One of them had coexistence of erythrocyte autoantibodies+alloantibodies, and the other two were only erythrocyte autoantibody positive. They did not show signs of hemolysis, were administered steroid and antihistamine, and none needed an additional transfusion.

The demographic data of the 78 patients who had a history of RBC suspension transfusion in our hospital previously and there were no erythrocyte autoantibodies, (Table I). 
The median increase (25\%-75\%) in pre- and post-transfusion HGB, HCT, and RBC was 1.1 (0.7-1.5)/ unit, 3.2 (1.8-4.1) / unit, and $0.4(0.2-0.5) /$ unit, respectively, in the periods when erythrocyte autoantibodies were not detected in the XM; and 1.1 (0.71.7) / unit, 3.1 (1.9-5.1) / unit, and 0.4 (0.2-0.6) / unit, respectively, in the periods when erythrocyte autoantibodies were detected in the $\mathrm{XM}$, with no statistically significant difference ( $p=0.27,0.13$ and 0.09 , respectively, Table II). There was no statistically significant difference according to gender ( $p=0.36,0.25$, and 0.16 , respectively, for females and 0.47 , 0.29 , and 0.33 , respectively, for males).

\section{DISCUSSION}

Blood transfusion stimulates the immune system and induces both erythrocyte alloantibody and autoantibody production. ${ }^{6,8}$ Patients who have undergone transfusion can show erythrocyte autoantibodies weeks and even months after the transfusion as some kind of delayed transfusion reaction. ${ }^{1,12}$ Ahrens et al. also reported that autoimmunisation was associated with alloimmunisation. ${ }^{2}$ There is a genetic predisposition in erythrocyte autoantibody development. ${ }^{7,9}$ Presence of erythrocyte autoantibodies indicates autoimmune pathologies are observed with the loss of immune tolerance to the body's tissues and should be investigated. .,12 $^{2}$

Many studies are reporting several rates of erythrocyte autoantibody positivity in the literature.$^{5-9,11}$ It is believed that determining the purposes of these differences and its reflections in clinical practice would be beneficial. ${ }^{7}$ In this study, the rate of erythrocyte autoantibodies positivity was $10.16 \%$ $(342 / 3,365)$. Erythrocyte autoantibody positivity rate alone or in combination with erythrocyte alloantibodies was $1.6 \%$ $(49 / 3044)$ in patients who selected polytransfused patient category, according to Cruz et al. ${ }^{13}$

The median age of these patients was $72 \pm 16.11$ (0-98 years) in this study (Table I). Several studies have shown that erythrocyte autoantibody formation was associated with advanced age with dramatic increasee in individuals aged over 50 years. ${ }^{8,14,15}$ In this study, $52 \%$ were males, $48 \%$ were females, $13 \%$ were Rh-negative, and $87 \%$ were Rh-positive. In the study by Koçyiğit et al., respective figures were $47 \%, 53 \%, 12 \%$, and $88 \% .^{8}$ It was reported that the erythrocyte autoantibody and alloantibody formation was more dominant in females than males. ${ }^{14,15}$

In this study, only $1 \%$ of the 342 patients had a reaction strength of +3 (Table I). The remaining $99 \%$ exhibited low positivity. However, false positives can be seen due to the incompatibilities in the techniques and kits used. This situation can be linked to the ability to detect even the weakest antibodies with low affinity with more sensitive techniques used today, and the importance of these techniques in the clinical setting is still under investigation. .,10,15 $^{-1}$

In this study, only eight of 342 patients evaluated by XM were incompatible by combining the recipient plasma and the donor RBC with anti-human-globulin. All alloantibodies were anti-Rh (three anti-E, three anti-c, one anti-e, one anti-C). In the 342 patients, erythrocyte alloantibody positivity was detected in $2.3 \%$ of the patients (8/342) and the coexistence of erythrocyte autoantibody+alloantibody in $0.24 \%$ of the patients $(8 / 3,365)$. According to a study by Makroo et al., the rate of erythrocyte alloantibody positivity was $0.49 \%$, and the rate of erythrocyte autoantibody+alloantibody coexistence was $0.05 \% .{ }^{11} \mathrm{~A}$ total of $64.1 \%$ of the detected erythrocyte alloantibody was anti-Rh, and the most frequently identified erythrocyte alloantibody was anti-E. Studies conducted with patients who frequently underwent transfusion and had erythrocyte autoantibody positivity have shown considerably different rates of comorbid erythrocyte alloantibody positivity. The positivity rate was $40 \%$ in a study by Blackall et al. and $28 \%$ in a study by Ahrens et al., whereas Datta et al. did not observe any erythrocyte alloantibody formation. ${ }^{2,6,14}$ In this study, eight patients, who had coexistence erythrocyte autoantibody+alloantibody, were again subjected to the serological XM with minor blood group-compatible RBC suspensions, and compatible RBC suspensions could be provided for all patients except for one patient. Compatible RBC suspensions could be provided in patients who had erythrocyte autoantibody positivity after XM, but these may not be effective in the patient plasma due to the weak reactivity of the autoantibodies. $^{14}$

Steroids, immunoglobulins, and plasmapheresis can remove erythrocyte autoantibodies. ${ }^{4}$ In this study, the first two options were used in three patients who had mild transfusion reaction. In the study by Koçyiğit et al., only one among the 17 patients, who had erythrocyte autoantibodies, developed a transfusion reaction and needed immunosuppressive therapy (steroid and rituximab) and an extra transfusion. ${ }^{8}$

In RBC suspensions transfusions for patients, who had erythrocyte autoantibody positivity, but XM was compatible and did not have in-vivo hemolysis, transfusion is generally well tolerated without causing any problems since the transfused erythrocytes have the same lifespan as autologous erythrocytes. ${ }^{1,4}$ On the contrary, erythrocyte autoantibodies were also reported to cause problems such as suppression of erythropoiesis and a lifespan shorter than normal in the transfused erythrocytes. ${ }^{1,6,12,16}$ Wheeler et al. reported that a complete response to 1 unit of RBC suspension was achieved with a $2 \%-3 \%$ increase in $\mathrm{HCT}(0.02-0.03)$ and a $1 \mathrm{~g} / \mathrm{dL}(10 \mathrm{~g} / \mathrm{L})$ increase in $\mathrm{HGB} .{ }^{17}$ In this study, the median increase (25\%-75\%) in pre- and post-transfusion HGB, HCT, and RBC in 78 patients who had a history of RBC suspension transfusion previously and there were no erythrocyte autoantibodies, which was detected in the serological XM applied in the previous transfusions, was 1.1 (0.7-1.7) / unit, 3.1 (1.9-5.1) / unit and $0.4(0.2-0.6) /$ unit, respectively, in the periods when erythrocyte autoantibodies were detected; however, there was no statistically significant difference between the periods when erythrocyte autoantibodies were detected and were not detected. There was also no statistically significance according to gender.

Transfusion centre, reporting erythrocyte autoantibody positivity to the clinic cause unnecessary confusion; especially in patients who are not incompatible XM. Erythrocyte autoanti- 
body positivity should always be evaluated and interpreted in relation to the clinical, presentation and laboratory data., ${ }^{48,19}$ Presence of erythrocyte autoantibodies becomes essentially important in case of incompatible XM, because it is desired that the allogeneic well shows lower reactivity than the $A C$ well, which is called "selection of the least incompatible". 1,20 Indeed, the most important issue in such patients is to check for the erythrocyte alloantibodies that are masked by the erythrocyte autoantibodies and can cause hemolytic transfusion reactions. ${ }^{1,2,4,6,12}$ Erythrocyte autoantibody detection is not actually a determinant of increased transfusion safety and even causes loss of time and higher costs. ${ }^{20-25}$ In a multicentre survey among the 40 regional centres, conducted by Liumbruno et al., it was reported that $A C$ in pre-transfusion screening (PTS) was used by one of 16 services of immunohaematology and transfusion medicine and two of 24 transfusion sections. It was emphasised that 35 of the 40 (88\%) transfusion structures do not plan to use the DAT or AC in PTS. The data also shows that 29 of 40 centres (73\%) performed DAT in patients with a positive indirect antiglobulin testing that is difficult to interpret. ${ }^{20}$ In a meta-analysis conducted by Ziman et al. consisting of 86 studies, $58 \%$ of respondents were performed DAT each time the indirect antiglobulin testing was positive. ${ }^{4}$

\section{CONCLUSION}

No statistically significant difference was found in terms of possible effects of erythrocyte autoantibodies on salient hemogram parameters. Erythrocyte autoantibodies positivity must be considered together with parameters such as previous transfusion history, other pre-transfusion laboratory test results, and clinical presentation and management.

\section{ETHICAL APPROVAL:}

The Ethics Committee of Balikesir University Clinical Researches approved this study (Number: 2019-148, Date: 23/10/2019).

\section{PATIENTS' CONSENT:}

As the study was designed retrospectively, data was collected from clinical archive after ethical approval.

\section{CONFLICT OF INTEREST:}

Authors declared no conflict of interest.

\section{AUTHORS' CONTRIBUTION:}

BA: Study design, data collection, literature review and writing. TKA: Drafting of work, data collection, statistical analysis.

$\mathrm{HD}, \mathrm{OB}$ : Data collection.

TE, EA: Writing-review and editing.

ES, BB: Did the literature search and final review.

All authors have critically reviewed the manuscript.

\section{REFERENCES}

1. ASCP, 2010. Educational commentary: Warm autoantibodies and transfusion. American Proficiency Institute 2010 1st Test Event.

2. Ahrens N, Pruss A, Kähne A, Kiesewetter H, Salama A. Coexistence of autoantibodies and alloantibodies to red blood cells due to blood transfusion. Transfusion 2007; 47(5):813-16.

3. Dameshek W, Levine P. Immunization with Rh factor in acquired haemolytic anaemia. N Engl J Med 1943; 228:641-4.

4. Ziman A, Cohn C, Carey PM, Dunbar NM, Fung MK, Greinacher $A$, et al. Warm-reactive (immunoglobulin G) autoantibodies and laboratory testing best practices: review of the literature and survey of current practice. Transfusion 2017; 57:463-77.

5. Kaur D, Bains L, Kandwal M, Parmar I. Erythrocyte alloimmunization and autoimmunization among blood donors and recipients visiting a tertiary care hospital. J Clin Diagn Res 2017; 11(3):EC12- EC15.

6. Datta SS, Mukherjee S, Talukder B, Bhattacharya P, Mukherjee $\mathrm{K}$. Frequency of red cell alloimmunization and autoimmunization in thalassemia patients: A report from Eastern India. Adv Hematol 2015; 2015:610931.

7. Elhence P, Solanki A, Verma A. Red blood cell antibodies in thalassemia patients in Northern India: risk factors and literature review. Indian J Hematol Blood Transfus 2014; 30:301-8.

8. Koçyiğit C, Eliaçık K, Kanık A, Atabay B, Türker M. Frequency of red cell allo- and autoimmunization in patients with transfusion-dependent beta thalassemia and affecting factors. Turk J Pediatr 2014; 56:487-92.

9. Domen RE. Warm red blood cell autoantibodies and the direct antiglobulin test revisited. Am J Clin Pathol 2004; 122:673-4.

10. Hannon JL. Management of blood donors and blood donations from individuals found to have a positive direct antiglobulin test. Transf Med Rev 2012; 26:142-52.

11. Makroo RN, Bhatia A, Hegde V, Chowdhry M, Thakur UK, Rosamma NL. Antibody screening \& identification in the general patient population at a tertiary care hospital in New Delhi, India. Indian J Med Res 2014; 140:401-5.

12. Pandey H, Das SS, Chaudhary R. Red cell alloimmunization in transfused patients: A silent epidemic revisited. Asian J Transfus Sci 2014; 8:75-7.

13. Cruz Rde O, Mota MA, Conti FM, Pereira RA, Kutner JM, Aravechia MG, et al. Prevalence of erythrocyte alloimmunization in polytransfused patients. Einstein (Sao Paulo) 2011; 9:173-8.

14. Blackall DP. Warm-reactive autoantibodies in pediatric patients: Clinical and serologic correlations. J Pediatr Hematol Oncol 2007; 29:792-6.

15. Petz LD, Garratty G. Blood transfusion in autoimmune hemolytic anemias. Immune hemolytic anemias, ed 2th, Philadelphia: Churchill Livingstone 2004;

16. Cerdas-Quesada C. Specificity of 136 patient's antibodies to human red blood cells in Dr. Max Peralta J Hospital Blood Bank 2004-February 2009. Transfus Apher Sci 2010; 42:105-08.

17. Wheeler CA, Calhoun L, Blackall DP. Warm reactive autoantibodies: Clinical and serologic correlations. Am J Clin Pathol 2004; 122:680-5.

18. Zantek ND, Koepsell SA, Tharp DR Jr, Cohn CS. The direct antiglobulin test: A critical step in the evaluation of hemolysis. Am J Hematol 2012; 87:707-9.

19. Worlledge SM. The interpretation of a positive direct antiglobulin test. BrJ Haematol 1978; 39:157-62.

20. Liumbruno GM, Tognaccini A, Bonini R, Curciarello G, Masini I, Ringressi $A$, et al. The role of the direct antiglobulin test in pre-transfusion investigations and the approach to selecting blood for transfusion in autoimmune haemolytic anaemia: results of a regional survey. Blood Transfus 2008; 6:156-62. 
21. Judd WJ, Butch SH, Oberman HA, Steiner EA,Bauer RC. The evaluation of a positive direct antiglobulin test in pretransfusion testing. Transfusion 1980; 20:17-23.

22. Judd WJ, Barnes BA, Steiner EA, Oberman HA, Averill DB, Butch $\mathrm{SH}$. The evaluation of a positive direct antiglobulin test (autocontrol) in pretransfusion testing revisited. Transfusion 1986; 26:220-4.

23. Stec N, Shirey RS, Smith B, Kickler TS, Ness PM. The efficacy of performing red cell elution studies in the pretransfusion testing of patients with positive direct antiglobulin tests. Transfusion 1986; 26:225-6.

24. Perkins JT, Arruza M, Fong K, Sosler SD, Saporito C. The relative utility of the autologous control and the antiglobulin test phase of the crossmatch. Transfusion 1990; 30:503-7.

25. Bator S, Litty C, Dignam C, Flynn JC Jr, Ballas SK. Current utilization of the direct antiglobulin test investigation: results of a hospital survey. Transfusion 1994; 34:457-8. 